\title{
AUTOMORPHISM GROUPS OF LAMINATED NEAR-RINGS DETERMINED BY COMPLEX POLYNOMIALS
}

\author{
by K. D. MAGILL, JR., P. R. MISRA and U. B. TEWARI
}

(Received 31st July 1981)

\section{Introduction}

The concept of a laminated near-ring was introduced in [2]. We recall briefly what it is. Let $N$ be a near-ring and let $a \in N$. Define a new multiplication on $N$ by $x * y=x a y$ for all $x, y \in N$. With this new multiplication and the same addition as before we have another near-ring which we denote by $N_{a}$. The near-ring $N_{a}$ is referred to as a laminated near-ring, the original near-ring $N$ is the base near-ring and $a$ is the laminator or laminating element.

In [2] the base near-ring $N$ was the near-ring of all continuous selfmaps of the reals under pointwise addition and composition and the laminator was an odd degree polynomial $P$. Our purpose in [2] was to describe the automorphism group Aut $N_{P}$ of the laminated near-ring $N_{P}$ and we were able to show that it is either $\mathbb{R}_{M}$ the multiplicative group of nonzero real numbers, $\mathbb{Z}_{2}$ the cyclic group of order two or the trivial group consisting of only one element. Moreover, it turned out that for most $P$, Aut $N_{P}$ is the trivial group.

In this paper our base near-ring is the near-ring $\mathscr{N}$ of all continuous selfmaps of the complex plane and the laminating element $P$ is any complex polynomial whatsoever. We will assume, however, that our polynomials are nonconstant. The problem of determining Aut $\mathscr{N}_{P}$ seems to be considerably more difficult than that of determining Aut $N_{P}$ [2]. Indeed the problem for complex polynomials is still far from being completely solved. For one thing, there are considerably more possibilities for Aut $\mathcal{N}_{P}$ than for Aut $N_{P}$.

In Section 2 we obtain some rather general results which follow from one of the main results in [1]. The near-rings $\mathscr{N}_{P}$ are treated in subsequent sections and all the results in those sections rely heavily on the results in Section 2. In Section 3, we characterise those complex polynomials $P$ for which Aut $\mathscr{N}_{P}$ is a finite group. In Section 4, we address ourselves to the problem of determining precisely what infinite groups can occur as Aut $\mathcal{N}_{P}$ and here we are able to completely solve the problem. There are exactly three such infinite groups and they are $G L(2)$, the full linear group of all $2 \times 2$ nonsingular real matrices, and two of its subgroups. Furthermore, for each of these infinite groups, we are able to completely characterise those polynomials $P$ such that Aut $\mathcal{N}_{P}$ is isomorphic to that particular group. 


\section{Some general results}

Let $X$ be a topological space, $G$ an additive topological group and $\alpha$ a continuous function from $G$ into $X$. The family $\mathcal{N}(X, G, \alpha)$ of all continuous functions from $X$ into $G$ forms a near-ring when addition of functions is defined pointwise and the product $f g$ of continuous functions $f$ and $g$ is defined by $f g=f \circ \alpha \circ g$. The near-ring $\mathscr{N}(X, G, \alpha)$ is referred to as a sandwich near-ring with sandwich function $\alpha$. For any topological group $G$ and any decomposition $\Pi$ of $G$ into mutually disjoint nonempty subsets, we let $T A(G, \Pi)$ denote the group, under composition, of all topological automorphisms $t$ of the group $G$ which satisfy $t[A] \in \Pi$ for each $A \in \Pi$. Now we are in a position to state our first result.

Theorem 2.1. Let $X$ be a completely regular Hausdorff space which has more than one point and contains a compact subspace with nonempty interior and let $G$ be a connected, locally arcwise connected metrizable topological group. Let $\alpha$ be a quotient map from $G$ onto $X$ and let $\Pi(\alpha)$ be the decomposition of $G$ which is induced by $\alpha$. Then Aut $\mathscr{N}(X, G, \alpha)$, the automorphism group of the sandwich near-ring $\mathscr{N}(X, G, \alpha)$, is isomorphic to the group $T A(G, \Pi(\alpha))$.

Proof. Let $\phi$ be any automorphism of $\mathcal{N}(X, G, \alpha)$. Then, according to Theorem (3.1) of [1] there exists a unique homeomorphism $h$ from $X$ onto itself and a unique topological automorphism $t$ in $T A(G, \Pi(\alpha)$ ) such that the following diagram commutes for each $f$ in $\mathcal{N}(X, G, \alpha)$.

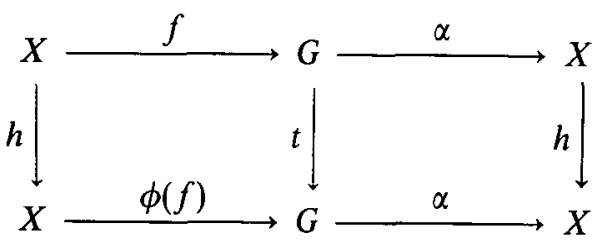

We refer to the pair $(h, t)$ as "the pair associated with $\phi$ " and we define a mapping $\Phi$ from Aut $\mathscr{N}(X, G, \alpha)$ into $T A(G, \Pi(\alpha))$ by $\Phi(\phi)=t$. Now let $\phi_{1}$ and $\phi_{2}$ be any two automorphisms of $\mathcal{N}(X, G, \alpha)$ with associated pairs $\left(h_{1}, t_{1}\right)$ and $\left(h_{2}, t_{2}\right)$ respectively. Then the triples $\phi_{1}, h_{1}, t_{1}$ and $\phi_{2}, \mathrm{~h}_{2}, \mathrm{t}_{2}$ both satisfy a commutative diagram of the form (2.1.1). It then follows that the following diagram also commutes

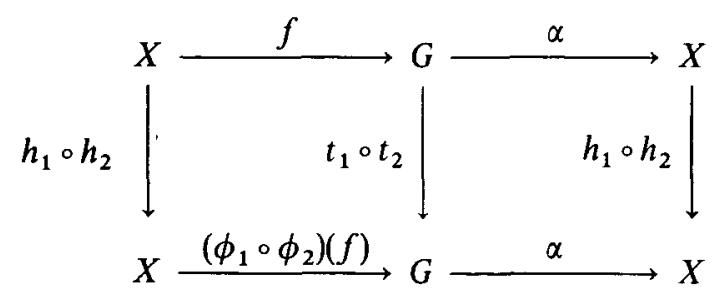

Thus, $\left(h_{1} \circ h_{2}, t_{1} \circ t_{2}\right)$ is the pair associated with $\phi_{1} \circ \phi_{2}$ and by uniqueness, it follows that 
$\Phi\left(\phi_{1} \circ \phi_{2}\right)=\Phi\left(\phi_{1}\right) \circ \Phi\left(\phi_{2}\right)$. In other words, $\Phi$ is a homomorphism. Suppose $\Phi(\phi)=e$, the identity automorphism. Then there exists a homeomorphism $h$ from $X$ onto $X$ such that the following diagram commutes.

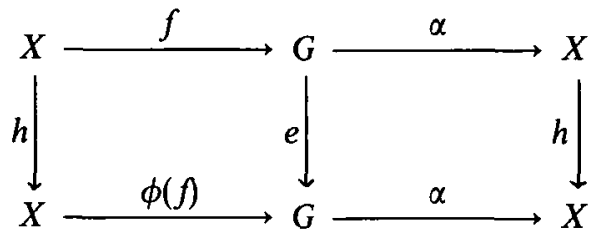

Thus, $h \circ \alpha=\alpha \circ e=\alpha$ and since the range of $\alpha$ is all of $X$, it follows that $h$ is the identity on $X$. Consequently, $\phi$ is the identity automorphism. This means that the kernel of $\Phi$ is trivial and that $\Phi$ is injective.

Now let $t$ be any element of $T A(G, \Pi(\alpha))$ and define a mapping $h$ as follows: let any $x$ in $X$ be given. Choose any $y \in G$ such that $\alpha(y)=x$ and definc

$$
h(x)=\alpha(t(y))
$$

Since $t[A] \in \Pi(\alpha)$ for each $A \in \Pi(\alpha)$ it readily follows that the definition of $h(x)$ does not depend on the choice of $y$. One notes immediately that

$$
h \circ \alpha=\alpha \circ t \text {. }
$$

Now suppose that $h\left(x_{1}\right)=h\left(x_{2}\right)$. Choose $y_{1}$ and $y_{2}$ so that $\alpha\left(y_{1}\right)=x_{1}$ and $\alpha\left(y_{2}\right)=x_{2}$. By (2.1.4) we have

$$
\alpha\left(t\left(y_{1}\right)\right)=h\left(\alpha\left(y_{1}\right)\right)=h\left(x_{1}\right)=h\left(x_{2}\right)=h\left(\alpha\left(y_{2}\right)\right)=\alpha\left(t\left(y_{2}\right)\right)
$$

Thus, $t\left(y_{1}\right)$ and $t\left(y_{2}\right)$ both belong to the same set in the decomposition $\Pi(\alpha)$. Since $t \in T A(G, \Pi(\alpha))$ it follows that $y_{1}$ and $y_{2}$ must belong to the same set in the decomposition $\Pi(\alpha)$. Consequently

$$
x_{1}=\alpha\left(y_{1}\right)=\alpha\left(y_{2}\right)=x_{2} .
$$

That is, $h$ is injective. To see that $h$ is surjective, let any $x \in X$ be given. Choose any $y$ such that $\alpha(y)=x$ and by (2.1.4) we get

$$
h\left(\alpha\left(t^{-1}(y)\right)\right)=\alpha\left(t\left(t^{-1}(y)\right)=\alpha(y)=x .\right.
$$

We have shown that $h$ is a bijection from $X$ onto $X$. Let $H$ be any open subset of $X$ and note that

$$
\alpha^{-1}\left[h^{-1}[H]\right]=(h \circ \alpha)^{-1}[H]=(\alpha \circ t)^{-1}[H] .
$$

Now $\alpha \circ t$ is continuous so $(\alpha \circ t)^{-1}[H]$ is open. Since $\alpha$ is a quotient map, this means 
that $h^{-1}[H]$ is open and hence that $h$ is continuous. Similarly, it follows from (2.1.4) that $h^{-1} \circ \alpha=\alpha \circ t^{-1}$ and we use this to get

$$
\alpha^{-1}[h[H]]=\left(h^{-1} \circ \alpha\right)^{-1}[H]=\left(\alpha \circ t^{-1}\right)^{-1}[H] .
$$

Since $\alpha \circ t^{-1}$ is continuous, $\left(\alpha \circ t^{-1}\right)^{-1}[H]$ is open and since $\alpha$ is a quotient map, $h[H]$ is open. This verifies the fact that $h$ is a homeomorphism. We now define an automorphism $\phi$ of $\mathcal{N}(X, G, \alpha)$ by $\phi(f)=t \circ f \circ h^{-1}$. One readily shows that $\Phi(\phi)=t$ and this completes the proof that Aut $\mathscr{N}(X, G, \alpha)$ is isomorphic to $T A(G, \Pi(\alpha))$.

In our next result, $V$ is a real topological vector space, $G(V)$ is its additive topological group and $T L A(V, \Pi)$ is the group, under composition, of all topological linear automorphisms $t$ of $V$ such that $t[A] \in \Pi$ for each $A \in \Pi$ where $\Pi$ is any decomposition of $V$ into mutually disjoint nonempty subsets.

Corollary 2.2. Let $X$ be a completely regular Hausdorff space which has more than one point and contains a compact subspace with nonempty interior, let $V$ be a metrizable real vector space and let $\alpha$ be a quotient map (in the topological sense) from $V$ onto $X$. Then Aut $\mathcal{N}(X, G(V), \alpha)$ is isomorphic to $T L A(V, \Pi(\alpha))$.

Proof. Since $V$ is metrizable, there is a metric for $V$ with the property that each sphere about 0 is circled. It is an easy exercise to show that such sets are arcwise connected so $V$ must be locally arcwise connected. Since any real topological vector space is arcwise connected, the hypothesis of Theorem 2.1 is satisfied so we conclude that Aut $\mathscr{N}(X, G(V), \alpha)$ is isomorphic to $T A(G(V), \Pi(\alpha))$ which certainly contains $T L A(V, \Pi(\alpha))$. To verify equality, let $t \in T A(G(V), \Pi(\alpha))$ and note that $t(r x)=r t(x)$ for any vector $x$ and any rational number $r$. This follows from the additivity of $t$ and it then follows that $t(k x)=k t(x)$ for any real number $k$ since $t$ is continuous. This completes the proof.

We conclude this section with one more corollary. It is the one to which we refer in later sections. Let $R^{N}$ denote the usual topological group of $N$-tuples of real numbers and let $L A\left(R^{N}, \Pi\right)$ denote the group, under composition, of all linear automorphisms $t$ of the real vector space $R^{N}$ which satisfy $t[A] \in \Pi$ for each $A \in \Pi$ where $\Pi$ is any decomposition of $R^{N}$. We then have

Corollary 2.3. Let $X$ be a completely regular Hausdorff space which has more than one point and contains a compact subspace with nonempty interior and let $\alpha$ be a quotient map from $R^{N}$ onto $X$. Then Aut $\mathcal{N}\left(X, R^{N}, \alpha\right)$ is isomorphic to $L A\left(R^{N}, \Pi(\alpha)\right)$.

Proof. This follows immediately from Corollary 2.2 and the fact that in a finite dimensional vector space, every linear automorphism is topological.

\section{Laminated near-rings determined by complex polynomials}

Throughout the remainder of the paper, we will be concerned with laminated nearrings of the type $\mathcal{N}_{P}$, that is, near-rings consisting of all continuous selfmaps of the 
complex plane $\mathscr{C}$ where addition is pointwise and multiplication is given by $f g=f \circ P \circ g$. Our goal, of course, is to determine Aut $\mathscr{N}_{P}$ for any polynomial $P$. In this paper we get a partial solution in that we are able to determine Aut $\mathscr{N}_{P}$ for certain $P$. As we mentioned before, however, we are far from having a complete solution to the problem.

In our main result in this section, we characterise those $P$ for which Aut $\mathcal{N}_{P}$ is a finite group. Before we proceed to the formal results, suppose we make a few observations about an arbitrary complex polynomial $P$. First of all, since $\mathscr{C}$ is an algebraically closed field, $P$ maps $\mathscr{C}$ onto $\mathscr{C}$ and $P$ is certainly a quotient map (in fact, $P$ is closed as well as open) so that we can apply Corollary 2.3 . It follows immediately that Aut $\mathcal{N}_{P}$ is isomorphic to $L A\left(R^{2}, \Pi(P)\right)$ which, hereafter, we will denote more simply by $L A(P)$. In other words, $L A(P)$ is the group of all linear automorphisms on the real vector space $\mathscr{C}$ with the property that $t[A] \in \Pi(P)$ whenever $A \in \Pi(P)$. We will not hesitate to use the fact that Aut $\mathcal{N}_{P}$ is isomorphic to $L A(P)$ without explicitly mentioning it. To assist us in proving the main result, it is convenient to have some lemmas.

Lemma 3.1. A linear automorphism $t$ of $\mathscr{C}$ belongs to $L A(P)$ if and only if for all distinct $z_{1}, z_{2} \in \mathscr{C}$, the following statements are equivalent

$$
\begin{gathered}
P\left(z_{1}\right)=P\left(z_{2}\right) \\
P\left(t\left(z_{1}\right)\right)=P\left(t\left(z_{2}\right)\right) .
\end{gathered}
$$

Lemma 3.2. Let $P$ be any polynomial on $\mathscr{C}$ and let $T$ be a polynomial whose degree is one. Then Aut $\mathscr{N}_{P}$ is isomorphic to Aut $\mathscr{N}_{\text {ToP }}$.

The proof of Lemma 3.1 is straightforward. As for Lemma 3.2, $T$ is a homeomorphism from $\mathscr{C}$ onto $\mathscr{C}$ so one can produce an isomorphism from $\mathscr{N}_{p}$ onto $\mathcal{N}_{T \circ P}$. Or, alternatively, one can notice that $\Pi(P)=\Pi(T \circ P)$ so that $L A(P)=L A(T \circ P)$.

Lemma 3.3. Let $\operatorname{Deg} P=n$. Then there exist at most $n-1$ complex numbers $\left\{r_{i}\right\}_{i=1}^{n-1}$ such that $P(z)-r_{i}$ has multiple roots.

Proof. Suppose, to the contrary, that there exist $n$ complex numbers $\left\{r_{i}\right\}_{i=1}^{n}$ such that $P(z)-r_{i}$ has multiple roots. For each $i$, let $z_{i}$ be a multiple root of $P(z)-r_{i}$. Then each $z_{i}$ is a root of $P^{\prime}(z)$ which is a contradiction since there are $n$ such $z_{i}$ and Deg $P^{\prime}=n$ -1 .

Lemma 3.4. Let $\operatorname{Deg} P=n$. Then there exist at most $n-1$ complex numbers $\left\{r_{i}\right\}_{i=1}^{n-1}$ such that $P^{-1}\left(r_{i}\right)$ contains less than $n$ points.

Proof. Suppose, to the contrary, that there exist $n$ such points $\left\{r_{i}\right\}_{i=1}^{n}$. Then each of the polynomials $P(z)-r_{i}$ has multiple roots and the previous lemma has been contradicted. 
Some remarks. We illustrate the previous lemma in the case of the cubic $P(z)=z^{3}$ $+a z^{2}+b z+c$. One can show, with a little calculation that if $a^{2}=3 b$, then $\Pi(P)$ contains exactly one singleton and the rest are triples. In this case, the singleton is $\{-(a / 3)\}$. If $a^{2} \neq 3 b$, then $\Pi(P)$ contains exactly two doubles and the rest are triples. In this case the doubles are

$$
\left\{\frac{-2 a+w}{6},-\frac{a+w}{3}\right\} \text { and }\left\{-\frac{2 a+w}{6},-\frac{a-w}{3}\right\}
$$

where $w=2 \sqrt{a^{2}-3 b}$.

We still need one more lemma. Its proof is quite straightforward and will be omitted.

Lemma 3.5. Let $P(z)=z^{2}+a z+c$ and let $z_{1}$ and $z_{2}$ be any two distinct complex numbers. Then $P\left(z_{1}\right)=P\left(z_{2}\right)$ if and only if $z_{1}+z_{2}+a=0$.

And now we are in a position to prove

Theorem 3.6. Let $P(z)=a_{n} z^{n}+a_{n-1} z^{n-1}+\cdots+a_{0}\left(a_{n} \neq 0\right)$ be a complex polynomial. Then Aut $\mathscr{N}_{\mathrm{P}}$ is a finite group if and only if $\operatorname{Deg} P \geqq 3$ and $a_{i} \neq 0$ for some $i \neq 0, n$.

Proof. (Sufficiency.) Suppose $\operatorname{Deg} P \geqq 3$ and $a_{i} \neq 0$ for some $i \neq 0, n$. We must show that Aut $\mathcal{N}_{P}$ is finite. There is one set in $\Pi(P)$ which will play an increasingly important role in our considerations and that is $P^{-1}(P(0))$. We will denote it by $Z(P)$. The reason that $Z(P)$ is important is because $0 \in Z(P)$ and since for any $t \in L A(P)$ we have $t(0)=0$ and $t[Z(P)] \in \Pi(P)$, it follows that $t$ must map $Z(P)$ bijectively onto itself. In this particular case where we are assuming $a_{i} \neq 0$ for some $i \neq 0$ or $n$, it follows that $Z(P)$ must contain at least one nonzero element $v$ (it may, in fact, contain only one nonzero element). If $Z(P)$ contains two linearly independent elements, we are through for each $t \in L A(P)$ must map $Z(P)$ bijectively onto itself and is completely determined by the values it takes on at those linearly independent elements. It follows that in this case, $L A(P)$ cannot have more than $(n-1)(n-2)$ elements.

It remains for us to consider the case where each pair of nonzero elements in $Z(P)$ is linearly dependent. Then, since any $t \in L A(P)$ maps $Z(P)$ bijectively onto itself, we must have $t(v)=k v$ for some nonzero real number $k$. Since $t\left(k^{m} v\right)=k^{m+1} v$ for all positive integers $m$, it follows that $k^{m} v \in Z(P)$ for all positive integers $m$. Since $Z(P)$ is finite we conclude that $k=1$ or $k=-1$ and we have

$$
t(v)=v \text { or } t(v)=-v \text { for each } t \in L A(P)
$$

Next, we assert that

there exists a nonzero real number $r$ such that $P^{-1}(P(r v))$ contains two linearly independent elements.

Deny (3.6.2). According to Lemma 3.4 there exists a nonzero real number $k_{1}$ such that 
$P^{-1}\left(P\left(k_{1} v\right)\right)$ consists of $n$ elements where $n$ is the degree of $P$. By our assumption, we have

$$
P^{-1}\left(P\left(k_{1} v\right)\right)=\left\{k_{1} v, k_{2} v, \ldots, k_{n} v\right\}
$$

where the $k_{i}$ are distinct real numbers. Define a real polynomial $Q$ by

$$
Q(x)=\left(x-k_{1}\right)\left(x-k_{2}\right) \ldots\left(x-k_{n}\right)
$$

and choose a real number $r_{1}$ such that $Q^{-1}\left(Q\left(r_{1}\right)\right)$ contains at most two real numbers and $P^{-1}\left(P\left(r_{1} v\right)\right)$ consists of $n$ elements. The former can be satisfied because of well known properties of real polynomials and the latter can be satisfied because of Lemma 3.4. Because we are denying (3.6.2), we have

$$
P^{-1}\left(P\left(r_{1} v\right)\right)=\left\{r_{1} v, r_{2} v, r_{3} v, \ldots, r_{n} v\right\}
$$

where the $r_{i}$ are distinct real numbers. Now from (3.6.3) we get

$$
P(z)=a_{n}\left(z-k_{1} v\right)\left(z-k_{2} v\right) \ldots\left(z-k_{n} v\right)+P\left(k_{1} v\right) .
$$

For any $r_{i} v \in P^{-1}\left(P\left(r_{1} v\right)\right)$, we then have

$$
\begin{aligned}
P\left(r_{1} v\right)=P\left(r_{i} v\right) & =a_{n}\left(r_{i} v-k_{1} v\right)\left(r_{i} v-k_{2} v\right) \ldots\left(r_{i} v-k_{n} v\right)+P\left(k_{1} v\right) \\
& =a_{n} v^{n}\left(r_{i}-k_{1}\right)\left(r_{i}-k_{2}\right) \ldots\left(r_{i}-k_{n}\right)+P\left(k_{1} v\right) \\
& =a_{n} v^{n} Q\left(r_{i}\right)+P\left(k_{1} v\right) .
\end{aligned}
$$

Since $a_{n} v^{n} \neq 0$, it follows immediately from (3.6.7) that $Q\left(r_{i}\right)=Q\left(r_{j}\right)$ for $1 \leqq i, j \leqq n$. In other words

$$
Q^{-1}\left(Q\left(r_{1}\right)\right)=\left\{r_{1}, r_{2}, \ldots, r_{k}\right\}
$$

consists of $n$ distinct real numbers. Since $\operatorname{Deg} P=n \geqq 3$, we have a contradiction since $r_{1}$ was chosen so that $Q^{-1}\left(Q\left(r_{1}\right)\right)$ contains no more than two real numbers. This contradiction allows us to conclude that statement (3.6.2) is indeed valid. Let $A$ $=P^{-1}(P(r v))$ and $B=P^{-1}(P(-r v))$ and let $t$ be any element in $L A(P)$. By (3.6.1), either $t[A]=A$ or $t[A]=B$. The linear automorphism $t$ is completely determined by the values it takes at the two linearly independent points of $A$ and there are only finitely many possibilities. In fact $2 n(n-1)$ is an upper bound for the number of elements in $L A(P)$ in this particular case.

(Necessity.) We accomplish this by showing that if $\operatorname{Deg} P<3$ then Aut $\mathcal{N}_{P}$ is infinite and similarly, if $a_{i}=0$ for $0<i<n$, then Aut $\mathscr{N}_{P}$ is also infinite. Suppose first that $\operatorname{Deg} P<3$. If $\operatorname{Deg} P=1$ then it is immediate that $L A(P)$ consists of all linear automorphisms of $\mathscr{C}$. Now consider the case where $P(z)=a z^{2}+b z+c(a \neq 0)$ and define 
$Q(z)=z^{2}+(b / a) z$. In view of Lemma (3.2) it is sufficient to show that Aut $\mathscr{N}_{Q}$ is infinite. Let $t$ be any linear automorphism of $\mathscr{C}$ such that $t(b / a)=b / a$. It is immediate that for any two distinct complex numbers $z_{1}$ and $z_{2}$, we have $z_{1}+z_{2}+b / a=0$ if and only if $t\left(z_{1}\right)$ $+t\left(z_{2}\right)+b / a=0$. Thus, Lemma 3.5 tells us that $Q\left(z_{1}\right)=Q\left(z_{2}\right)$ if and only if $Q\left(t\left(z_{1}\right)\right)$ $=Q\left(t\left(z_{2}\right)\right)$ and it now follows from Lemma 3.1 that $t \in L A(Q)$. Thus, $L A(Q)$ contains every linear automorphism of $\mathscr{C}$ which fixes $b / a$ and therefore must indeed be an infinite group.

It remains for us to show that Aut $\mathscr{N}_{P}$ is infinite when $P$ is of the form $P(z)=a z^{n}+b$. Here again, Lemma 3.2 assures us that it is sufficient to show that Aut $\mathscr{N}_{Q}$ is infinite where $Q(z)=z^{n}$. For a complex number $v \neq 0$, define a linear automorphism $t_{v}$ by $t_{v}(z)$ $=v z$. It is immediate that $Q\left(z_{1}\right)=Q\left(z_{2}\right)$ if and only if $Q\left(t_{v}\left(z_{1}\right)\right)=Q\left(t_{v}\left(z_{2}\right)\right)$ so that $L A(Q)$ contains all linear automorphisms of the form $t_{v}$ where $v \neq 0$. This concludes the proof.

\section{The infinite automorphism groups}

Let $G L(2)$ denote the full linear group of all real $2 \times 2$ non-singular matrices. Let $G_{1}$ denote the subgroup of $G L(2)$ consisting of all matrices of the form

$$
\left[\begin{array}{ll}
1 & a \\
0 & b
\end{array}\right] \text { where } b \neq 0
$$

and let $G_{c}$ denote the subgroup of $G L(2)$ consisting of all matrices of the form

$$
\left[\begin{array}{rr}
a & -b \\
b & a
\end{array}\right] \text { and }\left[\begin{array}{rr}
a & b \\
b & -a
\end{array}\right]
$$

where $a^{2}+b^{2} \neq 0$.

If Aut $\mathscr{N}_{P}$ is infinite, it must be one of these three groups. In this section, we characterise, for each of these groups, the polynomials $P$ such that Aut $\mathscr{N}_{P}$ is isomorphic to that particular group. We first derive a lemma (whose proof is somewhat computational) and several corollaries.

Lemma 4.1. Let $u+v i, x+y i$ and $x-y i$ be three vectors with the same absolute value such that $x \neq 0 \neq y$ and $u^{2} \neq x^{2}$. Let $t$ be any linear automorphism which maps these three vectors to vectors which also have the same absolute values. Then, there exists a nonzero complex number $w$ such that either $t(z)=w z$ for each $z \in \mathscr{C}$ or $t(z)=w \bar{z}$ for each $z \in \mathscr{C}$.

Proof. Let $t(1)=a+b i$ and $t(i)=c+d i$. Then

$$
t(x+y i)=x t(1)+y t(i)=(a x+c y)+(b x+d y) i
$$

and we get

$$
|t(x+y i)|^{2}=\left(a^{2}+b^{2}\right) x^{2}+\left(c^{2}+d^{2}\right) y^{2}+2 x y(a c+b d)
$$


and similarly,

$$
|t(x-y i)|^{2}=\left(a^{2}+b^{2}\right) x^{2}+\left(c^{2}+d^{2}\right) y^{2}-2 x y(a c+b d) .
$$

By equating (4.1.1) and (4.1.2) we immediately get $4 x y(a c+b d)=0$ which since $x \neq 0 \neq y$, implies that

$$
a c+b d=0
$$

It readily follows from (4.1.3) and the fact that $|t(u+v i)|^{2}=|t(x+y i)|^{2}$ that

$$
\left(a^{2}+b^{2}\right) u^{2}+\left(c^{2}+d^{2}\right) v^{2}=\left(a^{2}+b^{2}\right) x^{2}+\left(c^{2}+d^{2}\right) y^{2}
$$

which, in turn, implies

$$
\left(a^{2}+b^{2}\right)\left(u^{2}-x^{2}\right)=\left(c^{2}+d^{2}\right)\left(y^{2}-v^{2}\right)
$$

By hypothesis, $x^{2}+y^{2}=u^{2}+v^{2}$ and $u^{2} \neq x^{2}$ which, together with (4.1.5) implies that

$$
a^{2}+b^{2}=c^{2}+d^{2}
$$

From (4.1.3) we get $a^{2} c^{2}=b^{2} d^{2}$. We use this and (4.1.6) to get

$$
a^{2}\left(a^{2}+b^{2}-d^{2}\right)=a^{2} c^{2}=b^{2} d^{2}
$$

from which we get $a^{2}\left(a^{2}+b^{2}\right)=d^{2}\left(a^{2}+b^{2}\right)$. Now $a^{2}+b^{2} \neq 0$ since $t(1)=a+b i$ and $t$ is injective so we must have

$$
a^{2}=d^{2}
$$

One easily checks that (4.1.3) and (4.1.8) together imply that if $a \neq 0$ then either $a=d$ and $b=-c$ or $a=-d$ and $b=c$. If $a=0$ then $b$ may be either $c$ or $-c$. In any event, we have either $t(z)=w z$ or $t(z)=w \bar{z}$ where $w=t(1)=a+b i$.

Corollary 4.2. Let $t$ be a linear automorphism such that $|t(1)|=|t(i)|$ and $|t(x+y i)|=\mid t(x$ $-y i) \mid$ for some $x \neq 0 \neq y$. Then there exists a nonzero complex number $v$ such that $t(z)=v z$ for all $z \in \mathscr{C}$ or $t(z)=v \bar{z}$ for all $z \in \mathscr{C}$.

Proof. Let $t(1)=a+b i$ and $t(i)=c+d i$. Then $|t(x+y i)|=|t(x-y i)|$ implies $a c+b d=0$ and $|t(1)|=|t(i)|$ implies $a^{2}+b^{2}=c^{2}+d^{2}$. It follows from all this that the vectors $\sqrt{x^{2}+y^{2}}, x+y i$ and $x-y i$ satisfy the hypothesis of the previous lemma. This completes the proof.

By a circle in $\mathscr{C}$, we mean any set of the form $\{z:|z|=r\}$ where $r$ is a positive real number. We immediately get the following 
Corollary 4.3. A linear automorphism $t$ carries circles onto circles if and only if there exists a nonzero complex number $w$ such that $t(z)=w z$ for all $z$ or $t(z)=w \bar{z}$ for all $z$.

Now we are in a position to prove the main result of this section.

Theorem 4.4. Let $P$ be any complex polynomial. Then:

Aut $\mathcal{N}_{P}$ is isomorphic to $G L(2)$ if and only if $\operatorname{Deg} P=1$ or $P(z)=a z^{2}$ $+b$ where $a \neq 0$.

Aut $\mathcal{N}_{P}$ is isomorphic to $G_{1}$ if and only if $P(z)=a z^{2}+b z+c$ where $a \neq 0 \neq b$.

Aut $\mathscr{N}_{P}$ is isomorphic to $G_{c}$ if and only if $\operatorname{Deg} P \geqq 3$ and $P(z)=a z^{n}$ $+b$.

Proof. We first prove, in turn, the sufficiency portions of each of the three statements. If $\operatorname{Deg} P=1$, then it is immediate that every linear automorphism belongs to $L A(P)$ so that Aut $\mathscr{N}_{P}$ is indeed isomorphic to $G L(2)$. Now suppose $P(z)=a z^{2}+b$. Lemma 3.2 assures us that Aut $\mathscr{N}_{P}$ is isomorphic to Aut $\mathscr{N}_{Q}$ where $Q(z)=z^{2}$. For any linear automorphism $t$ and $z_{1}, z_{2} \in \mathscr{C}$ we have $Q\left(z_{1}\right)=Q\left(z_{2}\right)$ if and only if $Q\left(t\left(z_{1}\right)\right)=Q\left(t\left(z_{2}\right)\right)$ so that $L A(Q)$ consists of all linear automorphisms of $\mathscr{C}$. Hence, when $P(z)=a z^{2}+c$, Aut $\mathscr{N}_{P}$ is also isomorphic to $G L(2)$.

Now suppose $P(z)=a z^{2}+b z+c$ where $a \neq 0 \neq b$ and let $Q(z)=z^{2}+(b / a) z$. Again, Lemma 2.2 tells us that Aut $\mathcal{N}_{P}$ is isomorphic to Aut $\mathcal{N}_{Q}$. By Lemma 3.5, we have

$$
Q\left(z_{1}\right)=Q\left(z_{2}\right) \text { if and only if } z_{1}+z_{2}+(b / a)=0
$$

for distinct points $z_{1}$ and $z_{2}$ of $\mathscr{C}$. Let $t$ be any linear automorphism of $\mathscr{C}$ such that $t(b / a)=(b / a)$. It follows from (4.4.4) that for distinct $z_{1}, z_{2} \in \mathscr{C}$, we have $Q\left(z_{1}\right)=Q\left(z_{2}\right)$ if and only if $Q\left(t\left(z_{1}\right)\right)=Q\left(t\left(z_{2}\right)\right)$. Thus by Lemma 3.1, $t \in L A(Q)$. On the other hand, suppose $t \in L A(Q)$ and choose any two distinct points $z_{1}, z_{2}$ such that $Q\left(z_{1}\right)=Q\left(z_{2}\right)$. By Lemma 3.1, we must also have $Q\left(t\left(z_{1}\right)\right)=Q\left(t\left(z_{2}\right)\right)$ which by (4.3.4) means that the equalities

$$
z_{1}+z_{2}+(b / a)=0
$$

and

$$
t\left(z_{1}\right)+t\left(z_{2}\right)+(b / a)=0
$$

must hold simultaneously. This can only happen, of course, when $t(b / a)=(b / a)$. Thus $L A(Q)$ consists of all linear automorphisms which fix the vector $(b / a)$. It is an easy exercise to show that this group is isomorphic to $L A_{1}$ the group of all linear automorphisms which fix the vector 1 . For any $t \in L A_{1}$, we have $t(i)=a+b i$ where $b \neq 0$ and it follows that the mapping which sends $t$ to the matrix

$$
\left[\begin{array}{ll}
1 & a \\
0 & b
\end{array}\right]
$$

is an isomorphism from $L A_{1}$ onto $G_{1}$. 
Next, we consider the case where $\operatorname{Deg} P \geqq 3$ and $P(z)=a z^{n}+b$. Again, it is sufficient to show that Aut $\mathscr{N}_{Q}$ is isomorphic to $G_{c}$ where $Q(z)=z^{n}$. Let $H$ be the group of all linear automorphisms $t$ of $\mathscr{C}$ with the property that there exists a nonzero complex number $v$ such that either $t(z)=v z$ for all $z$ or $t(z)=v \bar{z}$ for all $z$. It follows from Lemma 3.1 that $H \subset L A(Q)$. On the other hand suppose that $t \in L A(Q)$ and $n \neq 4$. Let $w$ be an $n$th root of unity whose real and imaginary parts are both nonzero. Then $Q(1)=Q(w)=Q(\bar{w})$ which implies $Q(t(1))=Q(t(w))=Q(t(\bar{w}))$ which, in turn, implies $(t(1))^{n}=(t(w))^{n}=(t(\bar{w}))^{n}$. Thus, $|t(1)|$ $=|t(w)|=|t(\bar{w})|$ and it follows from Lemma 4.1 that $t \in H$. In the event $n=4$, we have $Q^{-1}(Q(1))=\{1,-1, i,-i\}$. Here we have $t(1)=v$ which means $Q^{-1}(Q(t(1)))=\{v,-v$, $i v$, $-i v\}$. Thus $t(i)$ is $i v$ or $-i v$ which implies $t(z)=v z$ or $t(z)=v \bar{z}$. We have shown that in any event $L A(Q)=H$. Now, for any $t \in H$, we have either $t(z)=v z$ or $t(z)=v \bar{z}$ for some $v \neq 0$. In the former case we define

$$
\phi(t)=\left[\begin{array}{rr}
a & -b \\
b & a
\end{array}\right]
$$

and in the latter we define

$$
\phi(t)=\left[\begin{array}{rr}
a & b \\
b & -a
\end{array}\right]
$$

where $a+b i$ is the vector $v$. One easily verifies that $\phi$ is an isomorphism from $H$ onto $G_{c}$.

We have now verified the sufficiency portions of (4.4.1), (4.4.2) and (4.4.3). In order to verify necessity, we need the fact that the three groups $G L(2), G_{1}$ and $G_{c}$ are mutually nonisomorphic. We will identify these groups with their corresponding groups of linear automorphisms when it is convenient to do so. First of all one verifies easily that

the squares of any two elements in $G_{c}$ commute

and with some calculation, one shows that the squares of $t$ and $l$ do not commute where

$$
t(x+y i)=(x+y)+y i \quad l(x+y i)=x+2 y i .
$$

Since both $t$ and $l$ belong not only to $G L(2)$ but also to $G_{1}$, it follows that $G_{c}$ cannot be isomorphic to either $G_{1}$ or $G L(2)$. As for $G_{1}$ and $G L(2)$ note that

$t \in G_{1}$ is an involution (has order two) if and only if $t(1)=1$ and $t(i)=a-i$ for some real number $a$.

It follows from (4.4.9) that the product of any three involutions in $G_{1}$ is again an involution so in order to conclude that $G L(2)$ and $G_{1}$ are not isomorphic, it is sufficient to produce three involutions of $G L(2)$ whose product, in some order, is not an involution. This is not difficult to do. For example $t_{1} \circ t_{2} \circ t_{3}$ is not an involution where $t_{1}(z)=-z, t_{2}(z)=\bar{z}$ and $t_{3}(x+y i)=y+x i$. In fact, one can show that $\left(t_{1} \circ t_{2} \circ t_{3}\right)^{2}(i)=-i$. 
Thus, we have established the fact that no two of the groups $G L(2), G_{1}$ and $G_{c}$ are isomorphic. Now we can establish the necessity portions of (4.4.1), (4.4.2) and (4.4.3). We take (4.4.1) first. Suppose that Aut $\mathscr{N}_{P}$ is isomorphic to $G L(2)$. Then by Theorem 3.6 we know that either $\operatorname{Deg} P<3$ or $a_{i}=0$ for all $i \neq 0, n$. We have already established that if $\operatorname{Deg} P \geqq 3$ and $a_{i}=0$ for all $i \neq 0, n$, then Aut $\mathscr{N}_{P}$ is isomorphic to $G_{c}$ and hence not to $G L(2)$. Thus we must have $\operatorname{Deg} P<3$. If $P(z)=a z^{2}+b z+c$ and $b \neq 0$, we know from previous considerations that Aut $\mathscr{N}_{P}$ is isomorphic to $G_{1}$ which is not isomorphic to $G L(2)$. Consequently when $P(z)=a z^{2}+b z+c$, we must have $b=0$. This means that the polynomial $P$ must either be of the form $a z+b$ or $a z^{2}+b$. This establishes the necessity of (4.4.1). The arguments for (4.4.2) and (4.4.3) follow in the same manner.

We remark, in conclusion, that much remains to be done in order to determine Aut $\mathscr{N}_{P}$ for an arbitrary complex polynomial $P$. To be sure, we have completely determined those infinite groups which occur as automorphism groups of the near-rings $\mathcal{N}_{P}$ but the problem of determining which finite groups can occur as Aut $\mathscr{N}_{P}$ has not been touched upon.

Acknowledgement. Most of the work for this paper was completed while the second author was on the faculty of Universidade Federal de Goias and the first and third authors were visiting there. The authors wish to take this opportunity to thank their friends and colleagues at the University for their kind hospitality and both the University and CNPq for their generous financial support.

\section{REFERENCES}

1. K. D. MAGiLl, JR., Semigroups and near-rings of continuous functions, General Topology and its Relations to Modern Analysis and Algebra, II, Proc. Third Prague Top. Symp., 1971 (Academia, 1972), 283-288.

2. K. D. MAGILL, JR., Automorphisms of laminated near-rings, Proc. Edinburgh Math. Soc. 23 (1980), 97-102.

State University of New York at Buffalo

Universidade Estadual de Campinas ItT KanPUR INDIA 